\title{
A note on antichains of words
}

\author{
James D. Currie* \\ Department of Mathematics and Statistics \\ University of Winnipeg \\ Winnipeg, Manitoba \\ Canada R3B 2E9 \\ currie@uwinnipeg.ca
}

Submitted: July 9, 1995; Accepted: October 14, 1995

\begin{abstract}
We can compress the word 'banana' as $x y y z$, where $x=$ 'b', $y=$ 'an', $z=$ 'a'. We say that 'banana' encounters yy. Thus a 'coded' version of yy shows up in 'banana'. The relation ' $u$ encounters $w$ ' is transitive, and thus generates an order on words. We study antichains under this order. In particular we show that in this order there is an infinite antichain of binary words avoiding overlaps.
\end{abstract}

AMS Subject Classification: 68R15, 06A99

Key Words: overlaps. antichains, words avoiding patterns

\section{Introduction}

The study of words avoiding patterns is an area of combinatorics on words reaching back at least to the turn of the century, when Thue proved [29] that one can find arbitrarily long words over a 3 letter alphabet in which no two adjacent subwords are identical. If $w$ is such a word, then $w$ cannot be written $w=x y y z$ with $y$ a non-empty word. In modern parlance, we would say that $w$ avoids $y y$. A word which can be written as $x y y z$ is said to encounter $y y$. Thue also showed that there are arbitrarily long words over a 2 letter alphabet avoiding yyy. One can quickly check that no word of length 4 or more over a 2 letter alphabet avoids yy. We say that yyy is avoidable on 2 letters, or 2-avoidable whereas yy is unavoidable on 2 letters. On the other hand, yyy is certainly 3-avoidable, because any word avoiding yy must avoid yyy also.

Bean, Ehrenfeucht and McNulty [4], and independently Zimin [30], characterized words which are avoidable on some large enough finite alphabet. If $p$ is a word over an $n$ letter alphabet, then

\footnotetext{
*This work was supported by an NSERC operating grant.
} 
THE ELECTRONiC JOURNAL OF COMBINATORICS 2 (1995), \#R21

$p$ is avoidable on some finite alphabet if $Z_{n}$ avoids $p$, where words $Z_{n}$ are defined recursively by

$$
\begin{aligned}
Z_{1} & =1 \\
Z_{n+1} & =Z_{n}(n+1) Z_{n}, n \in \mathbb{N} .
\end{aligned}
$$

Thus the pattern $a b c a c b$ is a pattern over a 3 letter alphabet, and is avoided by $Z_{3}=1213121$. It follows that $a b c a c b$ is avoidable on some large enough finite alphabet. The size of the smallest alphabet on which abcacb is avoidable isn't known. No avoidable pattern is known which is not 4-avoidable. The following conjecture is given by Baker [2]:

Conjecture 1.1 Every avoidable pattern is 4-avoidable.

The following problem has been open since 1979 [4]:

Problem 1.2 Find an algorithm which given a word $p$ determines the smallest $k$ such that $p$ is $k$-avoidable.

Cassaigne and Roth $[8,27]$ studied avoidable binary and ternary patterns $p$, giving when possible the smallest $k$ for which $p$ is $k$-avoidable. In such work, the most important patterns are the minimal ones; as discussed above, yyy is 3-avoidable because it contains yy. Similarly, if $w$ is 3-avoidable, then so is $w^{R}$, the reverse of $w$. It follows from the work of Cassaigne and Roth that a binary pattern is 2-avoidable exactly when it encounters one of $x x x, x y x y x, x y x x y$, xxyxyy, хухуyx and xxyyx. Consideration of minimal $k$-avoidable patterns leads to problems such as the following, posed in [3]:

Problem 1.3 Write $u \geq w$ if $u$ encounters $w$ or the reversal of $w$. This relation is a quasi-order, and factoring out the resulting equivalence relation gives a partial order. Let $\mu(w)$ be the size of the smallest alphabet on which $w$ is avoidable. For avoidable $w$, is there an infinite antichain on $\mu(w)$ letters such that each member of the antichain avoids $w$ ?

Perhaps the posing of this problem is too ambitious. An affirmative solution would imply that for any avoidable word $w$, it takes no more letters to avoid $w$ and $w^{R}$ simultaneously than it does to avoid $w$ alone. Strong evidence to the contrary is provided by the example $w=a b c a c b$. It seems likely that $a b c a c b$ is 2 -avoidable; there are words of length 1000 over $\{0,1\}$ avoiding $a b c a c b$. However, $a b c a c b$ and $b c a c b a$ are not simultaneously 2-avoidable. ${ }^{1}$

For this reason, it seems that a better question to ask is the following:

Problem 1.4 Write $u \geq w$ if $u$ encounters $w$. For avoidable $w$, is there an infinite antichain on $\mu(w)$ letters such that each member of the antichain avoids $w$ ?

This question was answered in the affirmative in the case where $w$ is 2-avoidable in [18] Note that for the sake of studying antichains it is unnecessary to move from the quasi-order to a partial order.

In a related paper [9] it was shown that for any $\epsilon>0$ there is an infinite antichain of such ternary words avoiding $x^{k}$ for $7 / 4<k<7 / 4+\epsilon$. Note that $7 / 4$ is the threshold of repetition

\footnotetext{
${ }^{1}$ Thanks to Kirby Baker for the use of his software which allowed the author to make these discoveries concerning abcacb.
} 
THE ELECTRONiC JOURNAL OF COMBINATORICS 2 (1995), \#R21

for words over a 3 letter alphabet; if $r<7 / 4$, we can find at most finitely many words over a 3 letter alphabet avoiding $x^{r}$.

The threshold of repetition for a 2 letter alphabet is 2. A word containing no subwords of the form $x^{k}$ for $k>2$ is called overlap-free. A word is overlap-free exactly when it avoids both the patterns $x x x$ and $x y x y x$.

In this note we prove that any binary word which avoids overlaps is an element of an infinite antichain of binary words avoiding overlaps.

\section{Preliminaries}

An alphabet $\Sigma$ is a set whose elements are called letters. A word $w$ over $\Sigma$ is a finite string of letters from $\Sigma$. The length of word $w$ is the number of letters in $w$, denoted by $|w|$. Thus $\mid$ banana $\mid=6$, for example. The language consisting of all words over $\Sigma$ is denoted by $\Sigma^{*}$. If $x, y \in \Sigma^{*}$, the concatenation of $x$ and $y$, written $x y$, is simply the string consisting of $x$ followed by $y$. The word with no letters is called the empty word and is denoted by $\epsilon$. Suppose $w \in \Sigma^{*}$. We call word $x$ a prefix of $w$ if $w=x y$, some $y \in \Sigma^{*}$. Similarly word $y$ is a suffix of $w$ if we can write $w=x y$, some $x \in \Sigma^{*}$. We call $y$ a subword of $w$ if we can write $w=x y z$, some $x, z \in \Sigma^{*}$. In the case that $x$ and $z$ are non-empty, $y$ is an internal subword of $w$.

Let $\Sigma, T$ be alphabets. A substitution $h: \Sigma^{*} \rightarrow T^{*}$ is a function generated by its values on $\Sigma$. That is, suppose $w \in \Sigma^{*}, w=a_{1} a_{2} \ldots a_{m} ; a_{i} \in \Sigma$ for $i=1$ to $\mathrm{m}$. Then $h(w)=h\left(a_{1}\right) h\left(a_{2}\right) \ldots h\left(a_{m}\right)$. A substitution is non-erasing if for every $a \in \Sigma,|h(a)| \neq \epsilon$.

Let $w, v$ be finite words over some alphabet $\Sigma$. We say that $w$ encounters $v$ if $w=x h(v) y$ for some non-erasing substitution $h: \Sigma^{*} \rightarrow \Sigma^{*}$. Otherwise we say that $w$ avoids $v$. If $x$ is a word we denote by $x^{n}$ the word consisting of $x$ repeated $n$ times in a row. Thus $x^{2}=x x, x^{3}=x x x$ and so on. We call a word $w$ a $k$-power if $w=x^{k}$, some $x \neq \epsilon$. A 2-power is also called a square. An overlap is a word of form $x x x$ or $x y x y x$ for some words $x$ and $y$. A word $w$ is $k$-power free if we cannot write $w=x y z$, where $y$ is a $k$-power. Thus $w$ is $k$-power free if $w$ avoids $x^{k}$. Similarly one speaks of square-free or overlap-free words.

An $\omega$-word over alphabet $\Sigma$ is an infinite sequence of letters of $\Sigma$. If $w=\left\{w_{i}\right\}_{i \in \mathbb{N}}$ is an $\omega$-word over $\Sigma$, then each finite initial segment $w_{1}, w_{2}, \ldots, w_{n}$ of $w$ will correspond to some word $w_{1} w_{2} \ldots w_{n}$ of $\Sigma^{*}$. In this case we say that $w_{1} w_{2} \ldots w_{n}$ is a prefix of $\omega$-word $w$. If $u$ is an $\omega$-word over $\Sigma$ we say that $u$ encounters $w$ if some finite prefix of $u$ encounters $w$. Otherwise, we say that $u$ avoids $w$.

We say that $w$ is $k$-avoidable if the set of words over $\Sigma$ avoiding $w$ is infinite, for some, hence for any, alphabet $\Sigma$ of size $k$. Equivalently, $w$ is $k$-avoidable if there is an $\omega$-word over an alphabet of size $k$ which avoids $w$. If $w$ is $k$-avoidable for some $k \in \mathbb{N}$ we say that $w$ is avoidable. Otherwise, $w$ is unavoidable. Let $S$ be a set of words. We say that $v$ avoids $S$ if $v$ avoids each $w \in S$.

Fix an alphabet $\Sigma$. The relation ' $w$ encounters $v$ ' is a quasi-order on $\Sigma^{*}$ which we will abbreviate by $w \geq v$. We will be interested in the quasi-ordered set $\left\langle\Sigma^{*}, \geq\right\rangle$.

Lemma 2.1 Suppose that $\mathcal{A} \subseteq \Sigma^{*}$ is an infinite antichain. Then there is an $\omega$-word over $\Sigma$ avoiding $\mathcal{A}$.

Proof: Let $\mathcal{A}=\left\{w_{i}\right\}_{i=1}^{\infty}$. If $w$ is a non-empty word, denote by $w^{\prime}$ the word obtained from $w$ by deleting the last letter. 
We claim that for each $i \in \mathbb{N}, w_{i}^{\prime}$ avoids $\mathcal{A}$ : If $v \leq w_{i}^{\prime}$ then $v \leq w_{i}$ by transitivity. Thus if $j \neq i$, then $w_{i}^{\prime}$ avoids $w_{j}$, because $w_{j}$ and $w_{i}$ are incomparable. On the other hand, since $w_{i}^{\prime}$ is shorter than $w_{i}$, certainly $w_{i}^{\prime}$ avoids $w_{i}$.

Since $\mathcal{A}$ is an infinite set of words over a finite alphabet, $\mathcal{A}$ contains arbitrarily long words. Thus the set $\mathcal{A}^{\prime}=\left\{w_{i}^{\prime}\right\}_{i=1}^{\infty}$ contains arbitrarily long words of $\Sigma^{*}$ avoiding $\mathcal{A}$. It follows by König's Infinity Lemma that there is an $\omega$-word over $\Sigma$ avoiding $\mathcal{A}$. $\square$

Lemma 2.2 Let $S$ be a finite set of avoidable words. Then there is an $\omega$-word over a finite alphabet avoiding $S$.

Proof: This is proved in $[4,30]$. Let $S=\left\{s_{i}: 1 \leq i \leq m\right\}$. For each $i$ pick $n_{i} \in \mathbb{N}$ and an $\omega$-word $w_{i}=\left\{w_{i j}\right\}_{j=1}^{\infty}$ over an alphabet $\Sigma_{i}$ of size $n_{i}$ avoiding $s_{i}$. Then the word $w=\left\{\left(w_{1 j}, w_{2 j}, \ldots, w_{m j}\right)\right\}_{j=1}^{\infty}$ over the alphabet $\prod_{i=1}^{m} \Sigma_{i}$ avoids $s$

To avoid $S$ it suffices to avoid a maximal antichain of minimal elements of $S$. Thus we could get by with a version of this lemma in which $S$ was restricted to be an antichain.

Corollary 2.3 Suppose that $\mathcal{A} \subseteq \Sigma^{*}$ is a finite antichain. Then there is an $\omega$-word over some finite alphabet avoiding $\mathcal{A}$.

Remark 2.4 It is striking that infinite antichains over finite sets are easier to avoid than finite antichains! That is, to avoid a finite antichain over $S$ it may be necessary to move to a larger alphabet, whereas this is not the case with infinite antichains. To give a concrete example, let $S$ be the set of all words of length 7 over $\Sigma=\{a, b\}$. Each word of $S$ is 2-avoidable, but any binary word of length 7 or more encounters an element of $S$.

An image of $x x x$ or $x y x y x$ under a non-erasing substitution is called an overlap. Note that a prefix of an overlap will be a square.

Theorem 2.5 There is an infinite antichain of binary words avoiding overlaps.

Proof: Following Thue [29], Define the map $h:\{a, b\}^{*} \rightarrow\{a, b\}^{*}$ by $h(a)=a b, h(b)=b a$. Let $l$ $=$ aabaab. Thus $l^{R}=$ baabaa. We see that the prefixes of $l$ which are suffixes of $l^{R}$ are exactly $a, a a, a a b a a$.

Lemma 2.6 Word $l$ is avoided by $h^{\omega}(a)$.

Proof: This was proved by Cassaigne [8, Section 2.6, Théorème 2.2].

Corollary 2.7 The word $l^{R}$, the reverse of $l$, is avoided by $h^{\omega}(a)$.

Let $n \in \mathbb{N}$. Then we can write $h^{2 n+2}(a)=a b b a b a a b u_{n} b a a b a b b a$ for some word $u_{n}$. Let $m_{n}=a a b a a b u_{n} b a a b a a$.

Remark 2.8 Word $l$ is a prefix and suffix of each $m_{n}$, but every internal subword of $m_{n}$ is a subword of $h^{\omega}(a)$. It follows that $l$ doesn't appear in $m_{n}$ internally. We note that for each $n$, $m_{n}$ is a palindrome.

Lemma 2.9 Let $n \in \mathbb{N}$. Then the word $m_{n}$ is overlap-free. 
THE ELECTRONiC JOURNAL OF COMBINATORICS 2 (1995), \#R21

Proof: Every internal subword of $m_{n}$ is a subword of $h^{\omega}(a)$, and is overlap-free. It remains to show that no prefix or suffix of $m_{n}$ is an overlap. As $m_{n}$ is a palindrome, we need only show that no prefix of $m_{n}$ is an overlap.

First note that no prefix of $m_{n}$ is a square of length 12 or greater; otherwise the prefix of $m_{n}$ of length 6 reappears internally, i.e. $m_{n}$ contains an $l$ internally, which is impossible. It follows that the shortest overlap which is a prefix of $m_{n}$ has length at most 11, and is a prefix of $m_{1}$. Inspection shows that no prefix of $m_{1}$ of length 11 or less is an overlap.

Theorem 2.10 The set $\left\{m_{n}\right\}_{n \in \mathbb{N}}$ is an antichain.

Proof: Let $i, j \in \mathbb{N}, i<j$. Clearly $m_{i}$ doesn't encounter $m_{j}$ since $m_{j}$ is longer than $m_{i}$. Suppose, for the sake of a contradiction, that $m_{j}$ encounters $m_{i}$. Say that $m_{j}=\alpha f\left(m_{i}\right) \beta$ where $f$ is a non-erasing substitution.

If $\alpha \neq \epsilon$ then an internal subword of $m_{j}$ encounters the prefix $l$ of $m_{i}$. Thus a subword of $h^{\omega}(a)$ encounters $l$. This is impossible by Lemma 2.6. Thus $\alpha=\epsilon$. Symmetrically, $\beta=\epsilon$.

Since $a$ is a prefix of $m_{i}, f(a)$ is a prefix of $m_{j}$. Thus $l$ and $f(a)$ are both prefixes of $m_{j}$, and one must be a prefix of the other. Since $a$ is an internal subword of $m_{i}, f(a)$ is an internal subword of $m_{j}$. Thus $l$ is not a prefix of $f(a)$. We conclude that $f(a)$ is a proper prefix of $l$. Symmetrically, $f(a)$ must be a proper suffix of $l^{R}$, the reverse of $l$. Thus $f(a)=a$, aa or aabaa. However, $a a$ is a subword of $m_{i}$, so that $f(a a)$ is a subword of the overlap-free word $m_{j}$. We conclude that $f(a)=a$.

We have so far determined $f(a)$. Now consider $f(b)$. Since $f\left(m_{i}\right)=m_{j}$ is longer than $m_{i}$, the length of $f(b)$ is greater than 1. Since $a a b$ and baa are subwords of $m_{i}$, both $a a f(b)$ and $f(b) a a$ are subwords of $m_{j}$. It follows that $f(b)$ has $b$ as a prefix and a suffix, since otherwise aaa appears in $m_{j}$, a contradiction since $m_{j}$ is overlap-free.

Since $a a b$ is a prefix of $m_{i}, f(a a b)=a a f(b)$ is a prefix of $m_{j}$. However, another prefix of $m_{j}$ is aabaab. Thus one of $f(b)$ and baab is a prefix of the other. Since $f(b)$ begins and ends with a $b$, we conclude that $b a a b$ is a prefix of $f(b)$. As $a a b$ appears internally in $m_{i}$, we conclude that $f(a a b)$ occurs internally in $m_{j}$. A prefix of $f(a a b)$ is $a a b a a b=l$. It follows that $l$ appears internally in $m_{j}$, and hence that $l$ is a subword of $h^{\omega}(a)$. This contradicts Lemma 2.6.

The supposition that $m_{j}$ encounters $m_{i}$ leads to a contradiction. We conclude that $m_{i}$ and $m_{j}$ are incomparable, and that in fact $\left\{m_{n}\right\}_{n \in \mathbf{N}}$ is an antichain.

\section{References}

[1] S. Aršon, Démonstration de l'existence des suites asymétriques infinies, Mat. Sb., (N.S.) 2, 769-779; Zbl. 18, 115.

[2] Kirby A. Baker, Some problems on avoidability. Exposé au LITP, 1992.

[3] Kirby A. Baker, George. F. McNulty \& Walter Taylor, Growth problems for avoidable words, Theoret. Comput. Sci. 69 (1989), no. 3, 319-345; MR 91f:68109.

[4] Dwight R. Bean, Andrzej Ehrenfeucht \& George McNulty, Avoidable Patterns in Strings of Symbols, Pacific J. Math. 85 (1979), 261-294; MR 81i:20075. 
THE ELECTRONiC JOURNAL OF COMBINATORICS 2 (1995), \#R21

[5] J. Brinkhuis, Non-repetitive sequences on three symbols, Quart. J. Math. Oxford Ser.(2) 34 (1983), 145-149; MR 84e:05008.

[6] T. C. Brown, Is there a sequence on four symbols in which no two adjacent segments are permutations of each other?, Amer. Math. Monthly 78 (1971), 886-888.

[7] Stanley Burris \& Evelyn Nelson, Embedding the dual of in the lattice of equational classes of semigroups, Algebra Universalis, 1 (1971/72), 248-253; MR 45 \#5257.

[8] Julien Cassaigne, Motifs évitables et régularité dans les mots, Thèse de Doctorat, Université Paris VI, Juillet 1994.

[9] Max Crochemore \& Pavel Goralcik, Mutually avoiding ternary words of small exponent, Internat. J. Algebra Comput. 1 (1991) 407-410.

[10] James D. Currie, Non-repetitive walks in graphs and digraphs, $\mathrm{PhD}$ thesis, University of Calgary (1987).

[11] James D. Currie, Which graphs allow infinite non-repetitive walks? Discrete Math. 87 (1991), 249-260; MR 92a:05124.

[12] James. D. Currie, Open problems in pattern avoidance, Amer. Math. Monthly 100 (1993), 790-793.

[13] James. D. Currie, Non-repetitive words: ages and essences, Combinatorica, to appear.

[14] F.M.Dekking,Strongly non-repetitive sequences and progression-free sets, J. Combin Theory Ser. A, 27 (1979), 181-185; MR 81b:05027.

[15] Françoise Dejean, Sur un théorème de Thue, J. Combin. Theory Ser. A 13 (1972), 90-99.

[16] Roger C. Entringer, Douglas E. Jackson \& J.A. Schatz, On non-repetitive sequences, J. Combin. Theory Ser. A 16 (1974), 159-164; MR 48 \#10860.

[17] Earl D. Fife, Binary sequences which contain no BBb, Trans. Amer. Math. Soc. 261 (1980), 115-136; MR 82a:05034

[18] Pavel Goralcik \& Tomas Vanicek, Binary Patterns in Binary Words, Internat. J. Algebra Comput. 1 (1991) 387-391.

[19] Andres. del Junco, A transformation with simple spectrum which is not rank one, Canad. J. Math. 29 (1977), 655-663; MR 57 \#6367.

[20] Jacques Justin, Characterization of the repetitive commutative semigroups, J. Algebra 21 (1972), 87-90; MR 46\#277.

[21] Juhani Karhumäki, On cube-free $\omega$-words generated by binary morphisms, Discrete Appl. Math. 5 (1983), 279-297; MR 84j:03081.

[22] Veikko Keränen, Abelian squares are avoidable on 4 letters, Automata, Languages and Programming: Lecture notes in Computer Sciences 623 (1992) Springer-Verlag 4152. 
THE ELECTRONiC JOURNAL OF COMBINATORICS 2 (1995), \#R21

[23] Filippo Mignosi, Infinite words with linear subword complexity, Theoret. Comput. Sci. 65 (1989), 221-242; MR 91b:68093.

[24] Marston Morse \& Gustav A. Hedlund, Symbolic dynamics I, II, Amer. J. Math. 60 (1938), 815-866; 62 (1940) 142; MR 1, 123d.

[25] P. S. Novikov \& S. I. Adjan, Infinite periodic groups I, II, III, Izv. Akad. Nauk. SSSR Ser. Mat. 32 (1968), 212-244;251-524;709-731;MR 39 \#1532a-c.

[26] P. A. B. Pleasants, Non-repetitive sequences, Proc. Cambridge Philos. Soc. 68 (1970), 267-274; MR 42 \#85.

[27] Peter Roth, Every binary pattern of length six is avoidable on the two-letter alphabet, Interner Bericht 6/89, Fachbereich Informatik, Universität Frankfurt.

[28] Robert. O. Shelton \& Raj P. Soni, Aperiodic words on three symbols I, II, III, J. reine agnew. Math. 321;327;330 (1981), 195-209;1-11;44-52; MR 82m:05004a-c.

[29] Axel Thue, Über unendliche Zeichenreihen, Norske Vid. Selsk. Skr. I. Mat. Nat. Kl. Christiana (1912), 1-67.

[30] A. Zimin, Blocking sets of terms, Mat. Sb. (N.S.) 119 (161) (1982); Math. USSR Sbornik 47 (1984), 353-364. 